\title{
Location and Sizing of Distributed Generation
}

\author{
Ajit P. Chaudhari ${ }^{1}$, Dr. E. Vijay Kumar ${ }^{2}$ \\ ${ }^{1}$ Ph.D. Scholar, ${ }^{2} H o D(E E \&$ EEE $)$ \\ ${ }^{1,2}$ RKDF Institute of Science \& Technology, Bhopal, India.
}

\begin{abstract}
This paper presents a review of the present work aimed to quantify the advantages of the location and sizing of distributed generation frameworks. In arrange to supply the growth of current and future electricity demand, attention is directed to distributed generation (DG). Distributed generation has gotten to be a complementary energy source for centralized generation, it has gained a lot of space in distribution systems. In expansion, the huge plants involve high costs, large greenhouse gas emissions and difficulty in obtaining environmental permits, these factors have also boosted the use of DG with renewable resources (wind, solar and water).
\end{abstract}

Keywords- Network Operators, Reactive Power Losses, Voltage Profiles, Active Power Losses, Centralized Generation,Distributed Generation.

\section{I -INTRODUCTION}

$\mathbf{E}$ lectric power systems are constantly growing due to the constant increase in consumption centers, mainly due to the increase in population . In addition, the emergence of large multinational companies, as well as the tightening of regulatory policies around the quality of service have contributed to the need to expand and operate power networks and power supply system efficiently. In this sense, network operators (NO) have been in need of improving operational characteristics in the electricity service, such as: improving voltage profiles, reducing active power losses (APL) and reactive power losses (RPL), reduce levels of non-served power and service quality indicators, among others.

Small-scale electricity generation, known as Distributed Generation (DG), is progressively common in distribution networks. This trend has been. inspired by various factors thathighlight the evolution of small-scale generation technologies and the preference for the use of renewable resources forelectricity generation.

The integration of distributed generation (DG) of adequate size and with optimal location can be used to obtain possible financial and technical advantages in the distribution networks. Therefore, the DG will provide power to the electrical systems to help meet current and future power needs in industrial, commercial and domestic fields. The incorporation of on-site production must be carried out carefully, as a transformation from passive networks to active networks is under way. Establishing an ideal connection point can lead to improvements such as: loss reduction, increase in reliability and reliability, and improvement of the tension profile. In this context, different authors have proposed methodologies to locate and / or properly size the DG that willbe integrated into the distribution networks. The proposed methodologies include analytical methods, mathematical programming, and Genetic algorithms, optimization based on artificial bee colonies. A common 


\section{International Journal of Innovations in Engineering and Science, www.ijies.net}

factor in studies of optimal location and dimensioning of DG is that only the point of view of a single agent is taken into consideration; either the operator of the distribution network or the investor in DG. Although many authors have approached the problem from a multi objective point of view such as, seeking to find a solution that meets several attributes at once; Very few have approached the problem from a muti-agent point of view. Examples of this last approach are presented. where bi-level programming is used to regulate only the optimal DG bond price. In both cases the problem of the worse level is supplanted by its optimality situations, resulting in an equivalent single level problem that can be solved by mathematical programming techniques.

\section{II- LOCATION AND OPTIMAL SIZING OF DISTRIBUTED GENERATION}

The use of a methodology based on optimization techniques should be able to examine the influence or effect of the DG on the characteristics of the DS, which can be very beneficial in the process of network planning and operation. The operating conditions to be improved and the set of restrictions tied to them must be modeled mathematically in order to achieve the resolution to the problem posed here, and then the selected optimization technique must be implemented. Next, the main components for the location and dimensioning of DG, the most used optimization techniques and the analysis of a set of works found in the literature are presented.

\subsection{Classification of AC and DC Buses}

In the previous paragraphs we have defined the technical conditions that can be improved when integrating DG, which can be converted into objective functions. These, together with the restriction system, form the mathematical model of the problem. An objective function is an expressed criterion through the variables that define the behavior of the system. This function can be maximized or minimized according to the needs of the problem. In the case of the integration of DG, it is possible that it belongs to the technical or economic aspects. The objective functions would be the following: Optimization techniques that implement this type of function deliver a set of optimal solutions, known as the Pareto optimal front. For the selection of any of the solutions offered, one must have the opinion of an expert in the subject (network operator) or use a decision-making technique. Usually this type of function is used when it is necessary to expand the methodical aspects of the network, with the lowest possible level of investment. This is where the conflicting objectives appear.

The mathematical model of the problem, in addition to having the objective function, must consider the restrictions that represent it. These have to be evaluated for each possible solution to the problem. Within the technical constraints in the DS, there are active and reactive power balances, voltage limits, power limits injected by the DG and transport capacity in the lines. It is worth noting that if any solution does not comply with the whole set of restrictions, it must be discarded or, failing that, a penalty must be proposed within the objective function.

\section{III -LITERATURE REVIEW}

When integrating DG into the distribution systems, it is necessary to be clear about the scenario where it will be worked. The test scenarios for the DG integration start from the different possible combinations between location, size, technology and number of DG units, which can be simple(one unit) or multiple (installation of two or more DG units). Depending on the type of scenario, a binary, continuous technique or a combination of both is required to solve the mathematical model described in previous section. In the literature there are various methods and techniques, whose classification and some studies performed are shown below.

\subsection{Analytical Methods}

They use a mathematical model as the representation of the system, evaluating it with a direct numerical solution, which can be developed by a commercial optimization software. They provide the benefit of having a short computational time, they are simple and precise. However, when the problemis complex, your solution is not as accurate. Different authors have applied this technique to the optimal integration problemof DG, such as those presented below.

Gözel and Hocaoglu present an approach for the location and sizing of DG, from a single generator. As an objective function, the minimization of total power losses was selected, and the use of operations with the admittance matrix or the Jacobian matrix was avoided. The method was tested in the IEEE systems of 12, 34 and 69 nodes, with a maximum loss reduction for each case of $45.41 \%, 55.34 \%$ and $59.09 \%$, respectively. This approach was compared with two othertechniques, and it was found that the response obtained by this was very close to the others. 


\section{International Journal of Innovations in Engineering and Science, www.ijies.net}

Gopiya et al., in 2015, presented an analytical approach for the integration of DG for the reduction of active and reactive powers in the network. Its methodology starts by looking for the level of active and reactive nodal power that most benefits the network, selecting as candidate nodes those that presented the greatest positive impact to the system. This methodology is used to locate one or several generators and was validated in the systems of 33 and 69 nodes, applying different test scenarios. The best results showed a reduction of $69.55 \%$ and $89.89 \%$.

Mahmoud et al., in 2016, they presented an approach for the optimal location of multiple DG units and an optimal flow of powers for their sizing. Different scenarios were implemented for the evaluation of the impact of DG. The technique was tested in the systems of 33 and 69 nodes, and a $65.5 \%$ reduction for the system of 33 nodes and $71.56 \%$ for the system of 69 nodes was found as the best solution among the different scenarios. It should be noted that much of the work reviewed for analytical methods does not examine the impact on the system's voltage profiles. Many authors do not do so because the power losses are closely related to the nodal tension profiles, so that if these are reduced, the stress profiles will increase. Care must be taken, as in some cases the tension profiles may exceed the upper limits established by norm.

\subsection{Numerical Methods}

They are also called calculation-based methods, and they are divided into two categories: direct and indirect methods. They are restricted when applying them to real problems, due to the high complexity they present; however, they are efficient in simple problems. To solve the DG problem in DS, the most commonly used approaches are linear programming ,non- linear programming, dynamic programming whole programming and stochastic programming.

Among the different works that exist in the literature is the one made by Khoa et al. , which presents an algorithm based on the primary double interior point technique to solve non- linear problems of optimal power flow. The key objective is to optimize the location and size of the DG to resolve the problem of reducing power losses. To authenticate their methodology they implement a system of 10 and 42 nodes. This article presents its results when analyzing the losses of each line.

In the study conducted by Kaur et al. Candidate nodes are initially preselected from a Combined Loss
Sensitivity [CLS] model. For optimum integration, the method of Sequential Quadratic Programming (SQP) and Branch and bound algorithm (B \& B) are used. The objective is to minimize system losses, based on peak loads. The proposed methodology was tested in the systems of 33 and 69 nodes, and matched with the techniques of exhaustive load flow (ELF), analytical improvement (Improved Analytical [IA]) and Particle Swarm Optimization (PSO). In the system of 33 nodes, a loss reduction of $86.1 \%$ is achieved, and two DG units are integrated, with the minimum injected power value (2765 MVA). For the 69 node system, the proposed method shows a better performance when integrating multiple DG units (with active and reactive power injection), with a loss reduction of $98.1 \%$. scenarios were evaluated, the first one proposes the injection from the node farthest from the feeder. It starts from an initial value of installed DG capacity. The maximum installed capacity of DG is $11.20 \mathrm{MW}$, located in nodes three and six. For the second scenario, without predetermining an initial location and size, there is a total generation of DG of $22.74 \mathrm{MW}$, distributed over the five nodes of the system. In this methodology, system losses are not taken into account as an objective function.

Keane et al. develop a methodology for the optimal location and sizing of DG system using linear programming approach. The interdependence of the nodes is used with respect to the set of constraints. The objective is to maximize the generation delivered from DG, taking into account the following restrictions: nominal current of the lines, capacity of transformers, maximum levels of short-circuit current in equipment, voltage limits in the nodes, renewable power limits available, among others The methodology was tested in a section of the distribution network of Ireland, consisting of feeder $(38 / 110 \mathrm{kV})$ and five nodes. Two scenarios were evaluated, the first one proposes the injection from the node farthest from the feeder. It starts from an initial value of installed DG capacity. The maximum installed capacity of DG is $11.20 \mathrm{MW}$, located in nodes three and six. For the second scenario, without predetermining an initial location and size, there is a total generation of DG of $22.74 \mathrm{MW}$, distributed over the five nodes of the system. In this methodology, system losses are not taken into account as an objective function.

\subsection{Heuristic and Metaheuristic Methods}

They are part of the set of non-exact methods, which despite not having mechanisms, which guarantee obtaining the optimal global solution, allow to obtain 


\section{International Journal of Innovations in Engineering and Science, www.ijies.net}

good solutions for problems of optimization of real life, with acceptable computational efforts. The computational effort involves two important aspects: the calculation time and the amount of memory required in the process. A heuristic is a simple procedure based on experience, good judgment or the solution of reduced mathematical models, which allows to find good quality solutions for complex problems of the non-polynomial complete type.

The heuristic methods are the simplest and lowest-level, and this means that they can fall into local optima, and thus move away from good solutions. For this reason, they are currently used as a starting point for metaheuristics or to reduce the solution space through the analysis of sensitivity indicators, since they allow identifying and selecting the most impactful set of attributes for one problem and discard the others. The most commonly used sensitivity indicators for heuristic techniques for DG integration are line overloads, nodal voltage limits, voltage stability index, among others .

Met heuristic techniques provides the path to change the procedures of subsidiary heuristics to achieve superior solutions efficiently, employing successful search strategies and bio inspired algorithms. Some of the most commonly used mono-objective techniques are: genetic algorithm, particle swarm optimization, simulated annealing, taboo search, immune algorithms, ant colony optimization and artificial bee colony algorithm, among others. Within the multi-objective techniques, the best known and most used are:non-dominated sorting genetic algorithm (NSGA) and the chaotic artificial bee colony (CABC) algorithm In the literature you can find a large number of works that implement this type of techniques in the integration of DG, among which are the following:

De Sousa et al .implement a sensitivity indicator for the reduction of candidate nodes to DG location, then apply a genetic algorithm (GA) for the location and dimensioning of DG, for which multiple generators are taken into account. The strategy is evaluated in a test system of 70 nodes and a network of 2678 nodes of a distribution company in Brazil. As an objective function, they take into account power losses, implementing an hourly demand curve. The authors show the application of the methodology to the case of 70 nodes, but they do not indicate the reduction of the level of power losses and the development in the stress profiles. In the case of the real system, they achieve a maximum loss reduction of $47 \%$, and in this way they carry out a broad analysis on the impact of the methodology on the system's voltage profile.
Kansal et al. present a methodology that allows to integrate three different types of DG in the DS, implementing as an objective function the losses of electrical power. They use the PSO algorithm as a solution technique. His work is evaluated through the use of the test systems of 33 and 69 nodes, which achieves a maximum reduction in power losses of $67.79 \%$ and $89.69 \%$, respectively. Additionally, they analyze the impact on the stress profiles and compare their technique with others such as the Artificial Bee Colony ( $\mathrm{ABC}$ ) and GA, applied in other publications. The injected power levels are higher than the slack node in the base case.

In the authors apply the technique of Ant Colony Optimization (ACO) for the optimal integration of DG, looking for the reduction in power losses. The methodology was validated in the IEEE systems of 12 and 15 nodes, with power losses of $84.66 \%$ and $75.68 \%$, respectively. Here, an analysis is not made on the impact on the voltage profiles, anda single generator with an active power injection close to the value of the one injected by the slack node is implemented. Mohandas et al. present a methodology for the integration of multiple DG units, using the Multiobjective Performance Index (MOPI), to improve the stability of the DS voltage. The problem is solved by implementing the Chaotic Artificial Bee Colony (CABC) algorithm. The efficiency of this approach is validated by the test systems of 38 and 69 nodes. In this work, only active power injection by the DG is considered, the model of constant power load, and scenarios are posed with other stress-dependent load models, such as domestic, industrial and commercial. This presents improvements in the stress indices for the different test scenarios. Among the cases analyzed above, the authors do not take into account the cost of the DGs.

\subsection{Hybrid Methods}

The hybrid methods allow the enhancement of met heuristic techniques by combining two or more of these, for the search for the solution of a problem. These methods provide a solution of good quality, close to or equal to the optimum. They are much implemented due to the characteristics of the problem discussed here, given that the location presents a binary characteristic and the dimensioning a continuous one. In most of the documents analyzed in the previous section, a met heuristic technique is applied for the location and an optimal flow for the dimensioning of the generators. This means that the dimensioning is tied to the 


\section{International Journal of Innovations in Engineering and Science, www.ijies.net}

objective function of the program that applies the flow, which usually takes into account only the reduction of power losses, which can limit the search to local optima. Among the works that are found in the literature that apply hybrid methods, we find the following:

Grisales et al. in they implement a GA for the location and the PSO algorithm for the sizing of three different types of DG technologies. They present a weighted multi-objective function, based on the power losses, the quadratic voltage error and the costs of the generators. To validate the methodology, the systems of 33 and 69 nodes were evaluated, with a reduction of power losses of $56.77 \%$ and $63.54 \%$, and the square error of tension of $80.49 \%$ and $82.08 \%$. An investment level is presented for each of the cases of USD \$ 1993667 and USD \$ 2386349, respectively. Kefayat et al. present a hybrid between the $\mathrm{ACO}$ and $\mathrm{ABC}$ algorithms, the first responsible for the location and the second dimensioning of the DG, which allows installing one to seven wind generators in the system. A weighted multi- objective function was implemented, which seeks the reduction of active and reactive power, emissions of $\mathrm{CO} 2$, investment costs and improvement of the voltage stability index. The uncertainty of the wind generation and the load is taken into account. To validate the methodology, the systems of 33 and 69 nodes were implemented, with different values in the weighting of the objective function. With this, several test scenarios were obtained for each case. The best solution was a reduction of $69.90 \%$ for power losses in the 33-node system with a seven-generator installation and a $71.33 \%$ reduction for the 69 -node system by installing three generators. Within the tables shown by the authors, the impact on the other objective functions can be analyzed; in all, positive impacts were obtained. Kansal et al. present a hybrid approach for the location of multiple DG units and implement different types of power injection to the system. For this, the PSO optimization technique and an analytical method for generator sizing were used. As an objective function, it presents the reduction of power losses in the DS. The technique was validated in the systems of 33 and 69 nodes, with which a maximum reduction was achieved between the different scenarios proposed of $94.45 \%$ and $98.1 \%$, respectively; there are too high levels of DG penetration, above the power produced by the slack node. In addition to this, the authors show that improvements are obtained in the voltage profiles and power factor of the system, indirectly.
It can be concluded from the analysis carried out, for the optimization methods and techniques proposed, that the objective functions most implemented in the literature are the reduction of power losses and the improvement of tension profiles. In general, the investment and operation costs of the generators are not included, which prevents an analysis of the recovery of the investment in a given period of time. It is worth noting the difficulty to recover the short-term investment in a DG project, only taking into account the reduction of power losses. It is recommended to analyze the economic incentives for the integration of this type of technology in the DS, given the improvement of the operating conditions and the reduction of $\mathrm{CO} 2$, offered by the regulators of each country.

The presentation of the computation times is not done in most documents, because when planning a distribution system, it is done for a long period (from five to ten years). This means that the computation time is not a critical variable for most authors, because these analyzes are not performed in real time. In the cases indicated, it is used to compare the efficiency between different optimization techniques. It must be said that one must be careful when comparing, since between one technique and another there is a wide difference between its complexity and the solution space analyzed.

\section{IV-FUTURE RESEARCH}

The trends in research to solve the problem of location and optimal dimensioning of DG point to the following issues:

- Coordinated planning: associated to the reconfiguration of the network through the simultaneous location of DG, capacitors, protection devices and power storage, considering a certain time, in search of improving the operating conditions of the system.

- Introduction of uncertainties to the mathematical model: they start from the knowledge of how the system behaves and include uncertain parameters, such as generation by renewable sources, variation of the load and prices of power. With the above, we are looking for a model that represents in a more realistic way the stochastic behavior of DS.

- Active management of the network: regarding the active operation of the distribution system, 


\section{International Journal of Innovations in Engineering and Science, www.ijies.net}

which implies the investment in communication and control systems that allow obtaining information in real time about the operation of the network and of the DG units that they are connected.

- Optimization methods: the new developments point to the creation of hybrids between the different techniques discussed here, in order to solve the problem of location (binary) and sizing (continuous), with the minimum computationaleffort (memory and time)

\section{V- CONCLUSION}

The integration of DG can be seen as an aid to the distributionsystem from two topics: it makes it possible to meet the increase in demand, using existing assets, and it can also positively impact the operational conditions of the network, provided that make a proper location and dimensioning of the generators. For its integration, the technical or economic need by the interested agent, the mathematical model of the problem and the optimization technique to be implemented must be taken into account. The interpretation of the results obtained depends on the model of the load used, since, according to the applied model, a specific time or a panorama of the time will be analyzed.

When integrating DG, the associated costs must be considered, in addition to the technical aspects. Within the articles reviewed here, it was found that most of the authors emphasis only on the technical issues of the network, regardless of the cost associated with the integration of DG (investment and operation), or the economic incentives provided by regulators. Both contexts are important when evaluating the rate of return on investment, which in many cases can define the viability of a project.

Many authors propose injecting high power levels through DG, sometimes above the power delivered by the main generator in the base case (without DG). This means that the solutions delivered by their methodologies cannot be taken to a real application. Therefore, the maximum DG capacity installed in the SD must be restricted, to the power demand plus the system losses. In addition to this, it is important to conduct a study of the power generation capacity for each type of DG technology located in the system. The most used test systems were 33 and 69 nodes, since they have high levels of power losses and nodal tensions violations; In addition, they allow us to appreciate more clearly the impact of the methodologies used. When conducting a review of the state of the art on the integration of DG, it was found that the test scenarios are born from the combination of their location and dimensioning, using as the best alternative the analysis of multiple units of DG. In general, the type of modeled load is mono-level and the most commonly used objective functions are the reduction of powerlosses and improvements in voltage profiles, with metaheuristic optimization techniques being the most implemented in recent years.

\section{REFERENCES}

[1] Gonçalves, Rogério R., John F. Franco, and MarcosJ. Rider. "Short-term expansion planning of radial electrical distribution systems using mixedinteger linear programming." IET Generation, Transmission \& Distribution 9, no. 3 (2014): 256266.

[2] Montoya, Oscar Danilo, Alejandro Grajales, Alejandro Garces, and Carlos Alberto Castro. "Distribution systems operation considering energy storage devices and distributed generation." IEEE Latin America Transactions 15, no. 5 (2017): 890900.

[3] Grisales, Luis Fernando, Alejandro Grajales, Oscar Danilo Montoya, Ricardo Alberto Hincapie, Mauricio Granada, and Carlos Alberto Castro. "Optimal location, sizing and operation of energy storage in distribution systems using multi-objective approach." IEEE Latin America Transactions 15, no. 6 (2017): 1084-1090.

[4] Aghaei, J., M. A. Akbari, A. Roosta, M. Gitizadeh, and T. Niknam. "Integrated renewable-conventional generation expansion planning using multiobjective framework." IET generation, transmission \& distribution 6, no. 8 (2012): 773-784.

[5] Hung, Duong Quoc, Nadarajah Mithulananthan, and R. C. Bansal. "Analytical expressions for DG allocation in primary distribution networks." IEEE Transactions on energy conversion 25, no. 3 (2010): 814-820.

[6] Kumar, Ashwani, and Wenzhong Gao. "Optimal distributed generation location using mixed integer non-linear programming in hybrid electricity markets." IET generation, transmission \& distribution 4, no. 2 (2010): 281-298.

[7] Zhao, Yuanyuan, Yiran An, and Qian Ai. "Research on size and location of distributed generation with vulnerable node identification in the active distribution network." IET Generation, Transmission \& Distribution 8, no. 11 (2014): 1801-1809. 


\section{International Journal of Innovations in Engineering and Science, www.ijies.net}

[8] Abu-Mouti, Fahad S., and M. E. El-Hawary. "Optimal distributed generation allocation and sizing in distribution systems via artificial bee colony algorithm." IEEE transactions on power delivery 26, no. 4 (2011): 2090-2101.

[9] Ren, Hongbo, Weisheng Zhou, Ken'ichi Nakagami, Weijun Gao, and Qiong Wu. "Multi-objective optimization for the operation of distributed energy systems considering economic and environmental aspects." Applied Energy 87, no. 12 (2010): 36423651.

[10] Shaaban, Mostafa F., Yasser M. Atwa, and Ehab F. El-Saadany. "DG allocation for benefit maximization in distribution networks." IEEE Transactions on Power Systems 28, no. 2 (2012): 639-649.

[11] López-Lezama, Jesús María, Antonio PadilhaFeltrin, Javier Contreras, and José Ignacio Muñoz. "Optimal contract pricing of distributed generation in distribution networks." IEEE transactions on power systems 26, no. 1 (2010): 128-136.

[12] Mobarakeh, Ashkan Sadeghi, Abbas RajabiGhahnavieh, and Aydin Zahedian. "A game theoretic framework for DG optimal contract pricing." In IEEE PES ISGT Europe 2013, pp. 1-5. IEEE, 2013.

[13] Georgilakis, Pavlos S., and Nikos D. Hatziargyriou. "Optimal distributed generation placement in power distribution networks: models, methods, and future research." IEEE transactions on power systems 28 , no. 3 (2013): 3420-3428.

[14] Ghosh, Sudipta, Sakti Prasad Ghoshal, and Saradindu Ghosh. "Optimal sizing and placement of distributed generation in a network system." International Journal of Electrical Power \& Energy Systems 32, no. 8 (2010): 849-856.

[15] Kansal, Satish, Vishal Kumar, and Barjeev Tyagi. "Optimal placement of different type of DG sources in distribution networks." International Journal of Electrical Power \& Energy Systems 53 (2013): 752760.

[16] Moradi, Mohammad Hasan, and M. Abedini. "A combination of genetic algorithm and particle swarm optimization for optimal DG location and sizing in distribution systems." International Journal of Electrical Power \& Energy Systems 34, no. 1 (2012) 66-74.

[17] Georgilakis, Pavlos S., and Nikos D. Hatziargyriou. "Optimal distributed generation placement in power distribution networks: models, methods, and future research." IEEE Trans. Power Syst 28, no. 3 (2013): 3420-3428.

[18] Rao, Singiresu S. Engineering optimization: theory and practice. John Wiley \& Sons, 2009.

[19] Mahmoud, Karar, Naoto Yorino, and Abdella Ahmed. "Optimal distributed generation allocation in distribution systems for loss minimization." IEEE Transactions on Power Systems 31, no. 2 (2016): 960-969.
[20] Kumawat, Manoj, Nitin Gupta, Naveen Jain, and Dipti Saxena. "Optimal distributed generation placement in power distributed networks: A review." In Electrical, Electronics, Signals, Communication and Optimization (EESCO), 2015 International Conference on, pp. 1-6. IEEE, 2015.

[21] Karthikeyan, S. Prabhakar, A. S. Harissh, K. Sathish Kumar, and I. Jacob Raglend. "A review on soft computing techniques for location and sizing of distributed generation systems." In Computing, Electronics and Electrical Technologies (ICCEET), 2012 International Conference on, pp. 163-167. IEEE, 2012.

[22] Wang, Caisheng, and M. Hashem Nehrir. "Analytical approaches for optimal placement of distributed generation sources in power systems." IEEE Transactions on Power systems 19, no. 4 (2004): 2068-2076

[23] Gözel, Tuba, and M. Hakan Hocaoglu. "An analytical method for the sizing and siting of distributed generators in radial systems." Electric Power Systems Research 79, no. 6 (2009): 912-918.

[24] Naik, Sevya Naik Gopiya, Dheeraj Kumar Khatod, and Mahendra Pal Sharma. "Analytical approach for optimal siting and sizing of distributed generation in radial distribution networks." IET Generation, Transmission \& Distribution 9, no. 3 (2014): 209220.

[25] Singh, Bindeshwar, V. Mukherjee, and Prabhakar Tiwari. "A survey on impact assessment of DG and FACTS controllers in power systems." Renewable and Sustainable Energy Reviews 42 (2015): 846-882.

[26] Porkar, S., P. Poure, A. Abbaspour-Tehrani-fard, and S. Saadate. "Optimal allocation of distributed generation using a two-stage multi-objective mixedinteger-nonlinear programming." European Transactions on Electrical Power 21, no. 1 (2011): 1072-1087.

[27] Khalesi, N., N. Rezaei, and M-R. Haghifam. "DG allocation with application of dynamic programming for loss reduction and reliability improvement." International Journal of Electrical Power \& Energy Systems 33, no. 2 (2011): 288-295.

[28] Foster, James D., Adam M. Berry, Natashia Boland, and Hamish Waterer. "Comparison of mixed-integer programming and genetic algorithm methods for distributed generation planning." IEEE transactions on power systems 29, no. 2 (2014): 833-843.

[29] Zhou, Zhe, Jianyun Zhang, Pei Liu, Zheng Li, Michael C. Georgiadis, and Efstratios $N$. Pistikopoulos. "A two-stage stochastic programming model for the optimal design of distributed energy systems." Applied Energy 103 (2013): 135-144.

[30] Khoa, Truong Quang Dang, P. T. T. Binh, and H. B. Tran. "Optimizing location and sizing of distributed generation in distribution systems." In Power 
Vol. 6, No. 10, 2021, PP. 188 -195

International Journal of Innovations in Engineering and Science, www.ijies.net

Systems Conference and Exposition, 2006. PSCE'06. 2006 IEEE PES, pp. 725-732. IEEE, 2006.

[31] Kaur, Sandeep, Ganesh Kumbhar, and Jaydev Sharma. "A MINLP technique for optimal placement of multiple DG units in distribution systems." International Journal of Electrical Power \& Energy Systems 63 (2014): 609-617.

[32] Keane, Andrew, and Mark O'Malley. "Optimal allocation of embedded generation on distribution networks." IEEE Transactions on Power Systems 20, no. 3 (2005): 1640-1646.

[33] Blum, Christian, Jakob Puchinger, Günther R. Raidl, and Andrea Roli. "Hybrid metaheuristics in combinatorial optimization: A survey." Applied Soft Computing 11, no. 6 (2011): 4135-4151.

[34] Prado, I. F., and L. P. Garces. "Chu-Beasley genetic algorithm applied to the allocation of distributed generation." In Innovative Smart Grid Technologies Latin America (ISGT LA), 2013 IEEE PES Conference On, pp. 1-7. IEEE, 2013.

[35] Sedighi, Mohsen, Arazghelich Igderi, and Amir Parastar. "Sitting and sizing of distributed generation in distribution network to improve of several parameters by PSO algorithm." In IPEC, 2010 Conference Proceedings, pp. 1083-1087. IEEE, 2010.

[36] Hussein, Dalia N., M. A. H. El-Sayed, and H. A. Attia. "Optimal sizing and siting of distributed generation." In Power Systems Conference, 2006. MEPCON 2006. Eleventh International Middle East, vol. 2, pp. 593-600. IEEE, 2006. 